\title{
Towards a parameter-free method for high Reynolds number turbulent flow simulation based on adaptive finite element approximation
}

\author{
Johan Hoffman $^{\mathrm{a}, \mathrm{b}, *}$, Johan Jansson ${ }^{\mathrm{a}, \mathrm{b}}$, Niclas Jansson ${ }^{\mathrm{c}}$, Rodrigo Vilela De Abreu ${ }^{\mathrm{a}}$ \\ ${ }^{a}$ Department of High Performance Computing and Visualization, KTH Royal Institute of Technology, 10044 Stockholm, Sweden \\ ${ }^{\mathrm{b}}$ Basque Center for Applied Mathematics, Mazarredo, 14. 48009 Bilbao, Basque Country, Spain \\ ${ }^{\mathrm{c}}$ RIKEN Advanced Institute for Computational Science, Kobe, Japan
}

Available online 29 December 2014

\section{Highlights}

- We present a parameter-free method for high Reynolds number turbulent flow.

- The method is based on adaptive mesh optimization using adjoint techniques.

- We connect the numerical approximation to dissipative weak solutions.

- Turbulent boundary layers are left unresolved, no boundary layer mesh is needed.

- We highlight benchmark problems for which the method is validated.

\begin{abstract}
This article is a review of our work towards a parameter-free method for simulation of turbulent flow at high Reynolds numbers. In a series of papers we have developed a model for turbulent flow in the form of weak solutions of the Navier-Stokes equations, approximated by an adaptive finite element method, where: (i) viscous dissipation is assumed to be dominated by turbulent dissipation proportional to the residual of the equations, and (ii) skin friction at solid walls is assumed to be negligible compared to inertial effects. The result is a computational model without empirical data, where the only model parameter is the local size of the finite element mesh. Under adaptive refinement of the mesh based on a posteriori error estimation, output quantities of interest in the form of functionals of the finite element solution converge to become independent of the mesh resolution, and thus the resulting method has no adjustable parameters. No ad hoc design of the mesh is needed, instead the mesh is optimized based on solution features, in particular no boundary layer mesh is needed. We connect the computational method to the mathematical concept of a dissipative weak solution of the Euler equations, as a model of high Reynolds number turbulent flow, and we highlight a number of benchmark problems for which the method is validated. The purpose of the article is to present the computational framework in a concise form, to report on recent progress, and to discuss open problems that are subject to ongoing research.
\end{abstract}

(c) 2014 Elsevier B.V. All rights reserved.

MSC: 65M60; 65M50

Keywords: Finite element method; Adaptive mesh refinement; Turbulence simulation

\footnotetext{
* Corresponding author at: Department of High Performance Computing and Visualization, KTH Royal Institute of Technology, 10044 Stockholm, Sweden.

E-mail addresses: jhoffman@kth.se (J. Hoffman), jjan@kth.se (J. Jansson), leifniclas.jansson@ @iken.jp (N. Jansson), rvda@kth.se (R.V. De Abreu).

URL: http://www.csc.kth.se/ hoffman (J. Hoffman).
} 


\section{Introduction}

In this paper we review our work towards a general framework for simulation of unsteady high Reynolds number turbulent flow using adaptive finite element methods, with the purpose to give a coherent presentation of the computational framework, to report on recent progress, and to highlight challenges and open problems. We can refer to the methodology as Direct Finite Element Simulation (DFS), and we recall the theoretical foundation and show how DFS offers solutions to the main challenges of turbulence simulation: how to assess the accuracy in a simulation, how to optimize the use of computational resources, and how to minimize the dependency on application specific model parameters.

Over the period 2010-2013, we have participated in a number of Computational Fluid Dynamics (CFD) benchmark workshops organized by the aerospace community (AIAA, NASA, DLR, Boeing, etc.), namely the BANC series [1-4] (Workshop on Benchmark Problems Airframe Noise Computation) and HiLiftPW-2 [5] (2nd AIAA CFD High Lift Prediction Workshop). These workshops have gathered leading research groups in the area, as well as major aerospace companies (e.g. Boeing, Cessna, Aerospace Kawasaki) and the main commercial software developers (e.g. ANSYS and Exa), with the objective to assess the state of the art computational methods for high Reynolds number aerodynamics and aeroacoustics in complex geometry. From the results presented in these workshop it is clear that our CFD methodology is unique in several aspects, in particular: (i) it was the only method that provided estimates of the error in the computation, (ii) it was the only method with automatic mesh adaptation, and (iii) it was the only method which was free from model parameters.

In the DFS framework, (i) and (ii) are achieved by goal-oriented a posteriori error estimation, where the error in a functional of interest can be expressed in terms of the residual of the approximate solution weighted by the solution of an associated adjoint (dual) problem. A general framework for a posteriori error estimation based on duality for differential equations was developed in the 1990s described, e.g., in [6-8] and the references therein. Our own work has been focused on the extension of this framework to CFD and turbulent flow [9-11]. The method is validated for a range of basic benchmark problems including flow past cylinders, spheres and squares [12-15], where the adaptive algorithm is shown to converge to the experimental reference data.

The parameter-free aspect (iii) of DFS is related to the fact that no subgrid scale stresses or Reynolds stresses are parametrized as in LES (Large Eddy Simulation) or RANS (Reynolds Averaged Navier-Stokes equations) [16], and that for high Reynolds numbers we assume that the skin friction of the boundary layers is dominated by inertial effects, so that a free slip boundary condition is used at solid boundaries [17-19]. In contrast to RANS and LES, no averaging or filtering operators are introduced in DFS, the resolution of the turbulent scales is determined as part of the computation, with the local mesh size adapted based on the required precision in the chosen quantity of interest. The motivation for the free slip boundary condition is based on the use of a mixed boundary condition, for the normal velocity and the tangential stresses, similar to standard wall layer models of LES [20].

We now proceed to put the DFS method into the context of research on computational methods for turbulence simulation, and mathematical research on the Navier-Stokes equations.

\subsection{Turbulence simulation}

Turbulence is central for our understanding of the world, including the geophysical flow of the ocean-atmosphere system, and the aerodynamics of vehicles, aircraft and wind turbines, for example. Simulation of turbulent flow is an outstanding problem that cuts through mathematics, numerical analysis, scientific computing and fluid mechanics. This interdisciplinary nature of the problem makes it particularly challenging.

The fundamental mathematical model of fluid flow is the nonlinear Navier-Stokes equations in 3 space dimensions, for which a proof of existence of classical (smooth) solutions in the general case is missing. To compute approximate solutions of the Navier-Stokes equations in the case of turbulent flow is extremely expensive; an heuristic argument estimates the number of degrees of freedom needed to resolve all turbulent scales in a Direct Numerical Simulation (DNS) to be of the order $R e^{9 / 4}$, with $R e$ the Reynolds number, which for many decades to come will make DNS impossible e.g. for geophysical flow or flight aerodynamics where $R e \gg 10^{6}$.

A reduction in the number of degrees of freedom is possible by instead considering models for averaged quantities, such as statistical mean fields in RANS or spatially filtered fields in LES, at the cost of introducing turbulence/subgrid 
models to model unresolved statistical fluctuations/subgrid scales [16]. This closure problem of turbulence modeling has proven to be an outstanding problem, where model parameters often have to be tuned to a particular application. Similarly, subgrid models introduce parameters that need to be chosen by the user, in particular near solid walls it is a challenge to select proper parameters to accurately capture phenomena such as flow separation [20].

In RANS/LES simulations, the relation between model errors and numerical errors is delicate, since the effect of the numerical method often is similar to the effect of turbulence/subgrid models, e.g. in the form of dissipation of kinetic energy. For example, the widely used Smagorinsky subgrid model [21] is closely related to methods of artificial viscosity used to stabilize numerical methods [22]. To tackle this issue, different approaches have been attempted, from using high order numerical methods with minimal dissipation, to interpreting the numerical stabilization as the subgrid model [23,11,24,25], or alternatively trying to balance the two sources of errors [26,10]. The relationship between the local resolution of the computational mesh and the turbulent length scales of RANS and LES also poses challenges, in particular near solid walls where small turbulent scales dictate a very fine mesh resolution which dominates the total computational cost [20].

In the context of finite element methods, Variational Multiscale Stabilized methods (VMS) [24] have been developed where the variational form of the Navier-Stokes equations is split into resolved scales and subgrid scales, by decomposing the underlying Hilbert space into a direct sum of a coarse space and a fine space, where a subgrid model is used to model the fine scale contribution.

\subsection{Dissipative weak solutions}

In the framework presented in this paper we follow a different path, where we avoid the process of averaging and scale separation altogether, and thus also the closure problem. For incompressible flow the mathematician Jean Leray in 1934 [27] proved the existence of a weak solution, or turbulent solution (solution turbulente) in the terminology of Leray, which is a function that satisfies a weak form of the Navier-Stokes equations (in the sense of distributions). The work of Leray laid the foundation for the modern mathematical theory of the Navier-Stokes equations, and the regularized form of the equations used by Leray later inspired a specific class of LES methods [28]. The question of uniqueness of a weak solution is still an open problem, and whether a weak solution is also a smooth (strong) solution is formulated as a $\$ 1$ million Clay Prize problem [29]. A weak solution does not satisfy the standard energy balance valid for a smooth solution of the Navier-Stokes equations, instead only an energy inequality can be shown to hold. Partial regularity of a weak solution was proven under the assumption that a generalized energy inequality holds [30,31], in which case the weak solution is referred to as a suitable weak solution. It can be shown that certain discrete approximations, including finite element approximations, converge to suitable solutions [32].

For a weak solution, dissipation without viscosity is possible. In particular, Duchon and Robert in 2000 introduced the notion of a dissipative weak solution as a model for high Reynolds number turbulent flow, where an explicit expression for the source of inviscid dissipation was derived, which took the form of a local lack of regularity in the velocity field [33]. Already in 1949, Onsager suggested that weak solutions of the inviscid Euler equations (assuming such exist) could be used to model high Reynolds number turbulent flow [34]:

"It is of some interest to note that in principle, turbulent dissipation as described could take place just as readily without the final assistance by viscosity. In the absence of viscosity, the standard proof of the conservation of energy does not apply, because the velocity field does not remain differentiable!"

A consequence of the idea that turbulent dissipation is independent of viscosity, is that it should be possible to model turbulence without any empirical parameters. Onsager also conjectured that in such "ideal" turbulence, the regularity of the velocity field could at most satisfy a Hölder condition with exponent $1 / 3$, otherwise the energy would be conserved. Several proofs of Onsager's conjecture have since then been presented, and in their 2006 review of the work of Onsager, Eyink and Sreenivasan end with a conclusion on the future of Onsager's ideas [35]:

"We believe that Onsager's theoretical vision of an ideal turbulence described by inviscid fluid equations is a proper idealization for understanding high Reynolds number flows. Needless to say, in real physical turbulence there is viscosity, which is always positive. [... I In the same way, the zero-viscosity limit, which supposes an infinite number of cascade steps, should be a good idealization for turbulence with a large but finite number of cascade steps, that is, a Reynolds number which is large but finite. The vindication of this belief, if it is true, must come from a set of calculational tools for the zero-viscosity limit, which will make it, in the end, a truly predictive device". 


\subsection{Direct finite element simulation}

In $[11,36,15]$ we show that approximate dissipative weak solutions can be computed to simulate turbulent flow using a stabilized finite element method that satisfies certain conditions on stability and consistency, which we refer to as a General Galerkin (G2) method. We also refer to the computational methodology as DFS, as an extension to turbulent flow of the general framework for a posteriori error estimation developed in the early 1990s [6-8]. In particular, for high Reynolds numbers we can set the viscosity to zero, thus realizing a computational model for turbulent flow free from empirical parameters.

We argue that the proper mathematical objects for assessing well-posedness in DFS are functionals of the computed weak solutions [37]. To represent the error in a functional of a dissipative weak solution we introduce a dual (adjoint) linearized problem, which opens for a posteriori estimation of the error in outputs of interest, such as the drag and lift of an airplane. The a posteriori error representation can be used to construct efficient adaptive algorithms, where the computational mesh and the finite element space are optimized for the computation of a particular output of interest (goal functional) to a certain accuracy using minimal computational resources. Thus also the local resolution of turbulent scales is determined as part of the computation (a posteriori) based on what functional is the goal of the simulation. For example, approximation of the aeroacoustic sources in a turbulent flow past a landing gear may demand a different mesh resolution than approximation of the aerodynamic forces on the same landing gear [1,2].

A classical problem of great significance is the prediction of aerodynamic forces acting on a body in a high Reynolds number flow. The choice of boundary conditions in the simulation is here central. As already noted, for realistic applications e.g. in flight aerodynamics, full resolution of a turbulent boundary layer in DNS is impossible due to the computational cost. In LES, typical models of the near wall turbulent flow are wall shear stress models [20] or local RANS models near the wall [38], where both approaches demand high resolution boundary layer meshes and calibration of model parameters.

In [18] we investigate a simple wall shear stress model parametrized by the skin friction stress generated by the turbulent boundary layer. We find that for very high Reynolds numbers, corresponding to small skin friction, the macroscopic flow is largely independent of the wall shear stress model. In particular, the skin friction parameter can be set to zero, corresponding to a free slip boundary condition, and no boundary layer mesh is needed. For high Reynolds number flow, DFS with a free slip boundary condition has been shown to be an efficient and accurate model for approximation of aerodynamic forces and aeroacoustic sources [1-3,5].

In this paper the G2/DFS framework is presented in Section 2, and we give a brief description of a detailed implementation of the method in Section 3. In Section 4 we summarize a set of validation studies for the computational model, in Section 5 we discuss challenges and open problems related to the dual problem, and in Section 6 we give a short summary.

\section{The G2/DFS computational framework}

Low Mach number flow can be approximated by incompressible flow, and the focus of this paper is the model of incompressible high Reynolds number turbulent flow around bluff or streamlined bodies, where the Reynolds number is defined as $R e=\frac{U L}{v}$, with $U$ a characteristic velocity, $L$ a characteristic length and $v$ is the kinematic viscosity. Further, we assume that turbulent boundary layers are fully developed, so that transition to turbulence in the boundary layers is not part of the model.

We choose mixed boundary conditions in the form of prescribing the normal component of the velocity and the tangential components of the viscous shear stresses, with the purpose to avoid resolution of thin boundary layers at high Reynolds numbers. The normal velocity is set to zero relative the body, corresponding to a no penetration boundary condition, whereas the tangential shear stresses must be provided as data to the problem. Boundary stress data is typically not available, but viscous shear stresses, measured in the form of a non-dimensional skin friction coefficient, is known to decrease with increasing Reynolds number [39]. In LES, wall-layer models take a similar form, with wall shear stress proportional to skin friction [20]. Thus, this form of boundary conditions can be motivated both by standard arguments from the LES literature [16], noting that both LES and DFS concern models of the unresolved turbulent flow near the boundary, and by strict mathematical arguments choosing mixed Dirichlet-Neumann type boundary conditions for the Navier-Stokes equations. 


\subsection{Mathematical model}

The Navier-Stokes equations for incompressible flow of (small positive) constant kinematic viscosity $v$ in a (bounded) volume $\Omega$ in $\mathbf{R}^{3}$ around a fixed rigid body with smooth boundary $\Gamma$ over a time interval $I=[0, T]$, take the form: Find the velocity $u=\left(u_{1}, u_{2}, u_{3}\right)$ and pressure $p$ depending on $(x, t) \in \Omega \cup \Gamma \times I$, such that

$$
\begin{aligned}
& \dot{u}+(u \cdot \nabla) u+\nabla p-\nabla \cdot \tau=0 \quad \text { in } \Omega \times I, \\
& \nabla \cdot u=0 \quad \text { in } \Omega \times I, \\
& u_{n}=0 \quad \text { on } \Gamma \times I, \\
& \tau_{s_{k}}=\beta u_{t_{k}} \quad \text { on } \Gamma \times I, \\
& u(\cdot, 0)=u^{0} \quad \text { in } \Omega,
\end{aligned}
$$

with $k=1,2, \dot{u}=\frac{\partial u}{\partial t}, u_{n}=u \cdot n$ the fluid velocity normal to $\Gamma$ with $n$ a unit outward normal vector, $\tau=$ $\tau(u)=2 v \epsilon(u)$ the viscous stress with $\epsilon(u)$ the standard velocity strain, $\tau_{s_{k}}$ the tangential stresses and $u_{t_{k}}=u \cdot t_{k}$ the tangential velocities with $t_{k}$ orthogonal tangent vectors, and $u^{0}$ a given initial condition.

We assume suitable far-field inflow/outflow boundary conditions, and we note the Dirichlet-Neumann boundary condition in the normal and tangential directions, respectively, with the effect of turbulent boundary layers modeled by a (skin) friction parameter $\beta$. With zero viscosity $v$ and boundary friction $\beta$, the model (1) reduces to the inviscid Euler equations.

In [33] it is shown that for any weak solution $(u, p)$ of the Euler equations (assumed to exist), a local energy equation is satisfied (in the sense of distributions)

$$
\frac{d}{d t}\left(\frac{1}{2}|u|^{2}\right)+\nabla \cdot\left(u\left(\frac{1}{2}|u|^{2}+p\right)\right)+D(u)=0,
$$

with $D(u)$ defined in terms of the local smoothness of $u$. If $D(u) \geq 0$, then $(u, p)$ is referred to as a dissipative weak solution.

\subsection{Finite element method}

In G2/DFS we compute approximate solutions of the Navier-Stokes equations (1) by a weighted residual stabilized finite element method, of the form: Find $\hat{U}=(U, P) \in V_{h}$ such that for all $\hat{v}=(v, q) \in V_{h}$

$$
[R(U ; \hat{U}), \hat{v}]+[h R(U ; \hat{U}), R(U ; \hat{v})]=0,
$$

where $V_{h}$ is a space-time finite element space with velocities $v$ satisfying $v \cdot n=0$ on $\Gamma$, $[\cdot, \cdot]$ is an $L_{2}(\Omega \times I)$ inner product, $R(U ; \hat{v})=(\dot{v}+U \cdot \nabla v+\nabla q, \nabla \cdot v)$ is the residual, and $h$ is the local mesh size. The first term in (3) establishes $\hat{U}$ as a weak solution of (1) and the second term introduces kinetic energy dissipation

$$
D_{h}(\hat{U})=[h R(U ; \hat{U}), R(U ; \hat{U})]=\left\|h^{0.5} R(U ; \hat{U})\right\|_{L_{2}}^{2}
$$

bounded by data with $\|\cdot\|_{L_{2}}$ an $L_{2}(\Omega \times I)$ norm [36].

Notice that here $v$ and $\beta$ are set to zero with instead the weighted residual stabilization introducing a dissipative effect as an automatic turbulence model. This is analogous to the dissipative weak solutions introduced by Duchon and Robert [33], with dissipation caused by a lack of smoothness in the velocity field, unrelated to viscous dissipation. For a smooth solution $\hat{U}$, corresponding to a small residual in $L_{2}(\Omega \times I)$, the dissipative effect of the weighted residual stabilization vanishes. In Section 4 we recall validation results that show that DFS based on the Euler equations is a good approximation of high Reynolds number turbulent flow. We also find that with sufficient mesh resolution, $D_{h}(\hat{U})$ in (4) is independent of the local mesh size $h$ [15].

In Section 3 we give a precise definition of a particular implementation of G2/DFS, but many choices are possible as long as certain conditions for stability and consistency are satisfied [36,15]. 


\subsection{Boundary conditions}

It is well known that for viscous flow, thin boundary layers develop near solid walls over which the velocity changes from the velocity of the wall to the free stream velocity. The thickness of these boundary layers scale with the Reynolds number, and at high Reynolds numbers the boundary layers are so thin that computational resolution is impractical or impossible. At sufficiently high Reynolds numbers the boundary layers undergo transition to turbulence, where computational resolution of turbulent boundary layers is even less realistic in any application of significance [20]. For example, full computational resolution in DNS of the turbulent flow around a car or an airplane, including boundary layers, is impossible for many decades to come [40].

Ever since the introduction of the boundary layer concept by Prandtl [41], models of the flow decoupling the boundary layer from the interior flow have been pursued, in which the effect of the boundary layer is modeled as a boundary condition for the interior flow [20]. In (1) we model the effect of turbulent boundary layers as a small skin friction stress at the solid boundary, which dissipates kinetic energy proportional to $\beta$. The sensitivity of the solution with respect to perturbations in the skin friction parameter $\beta$ is investigated in [17,18], where it is found that for small $\beta$ (corresponding to high Reynolds numbers) the sensitivity is low. In particular, for external flow at Reynolds numbers higher than $R e>10^{6}$ we typically approximate the small skin friction by zero, which makes DFS into a parameter-free model of high Reynolds number external flow.

We have presented evidence in the form of analysis and computational studies, that the macroscopic features of high Reynolds number flow, including 3D flow separation, is mainly determined by large scale stability aspects, rather than boundary layer effects $[19,1-3,5]$.

\subsection{A posteriori error estimation}

The goal of a simulation can often be formulated as computing a number of output quantities, for example the lift and drag of an airplane. To estimate the error in an output functional of interest we use a posteriori error analysis based on sensitivity information from the solution of a adjoint (dual) problem.

We define a target functional $M(\hat{u})=[\hat{u}, \hat{\psi}]+\left[p n, \psi_{\Gamma}\right]_{\Gamma}$, where $\hat{\psi}=(\psi, \chi)$ is a given weight function acting as data for the dual problem, and $\psi_{\Gamma}$ is boundary data for the dual velocity, with $[\cdot, \cdot]_{\Gamma}$ a $L_{2}(\Gamma \times I)$ inner product. For example, to choose the sum of the lift and drag of an airplane as the target functional we set $\hat{\psi}=0$ and $\psi_{\Gamma}=v_{D}+v_{L}$, with $v_{D}$ and $v_{L}$ unit vectors in directions opposite and normal to the flight direction, respectively.

The difference in output $M(\hat{u})-M(\hat{U})=[\hat{u}, \hat{\psi}]+\left[p n, \psi_{\Gamma}\right]_{\Gamma}-[\hat{U}, \hat{\psi}]-\left[P n, \psi_{\Gamma}\right]_{\Gamma}$ of two DFS solutions $\hat{u}$ and $\hat{U}$ on different meshes with maximal mesh size $h$, can be represented as

$$
M(\hat{u})-M(\hat{U})=[R(u ; \hat{u})-R(U ; \hat{U}), \hat{\varphi}]
$$

where $\hat{\varphi}=(\varphi, \theta)$ is a solution of the adjoint linearized problem

$$
\begin{aligned}
& -\dot{\varphi}-(u \cdot \nabla) \varphi+\nabla U^{T} \varphi+\nabla \theta=\psi \quad \text { in } \Omega \times I, \\
& \nabla \cdot \varphi=\chi \quad \text { in } \Omega \times I, \\
& \varphi=\psi_{\Gamma} \quad \text { on } \Gamma \times I, \\
& \varphi(\cdot, T)=0 \quad \text { in } \Omega,
\end{aligned}
$$

where $\left(\nabla U^{T} \varphi\right)_{j}=\sum_{i=1}^{3} \partial U_{i} / \partial x_{j} \varphi_{i}$.

If we assume that the time step is bounded by some constant times $h$, we can bound the output error using basic analysis [36]:

$$
|M(\hat{u})-M(\hat{U})| \leq C\left\|h^{0.5} \hat{\varphi}\right\|_{H^{1}},
$$

with a constant $C$ depending on data and bounds on the magnitude of the discrete velocities $u$ and $U$. We can thus guarantee stability in output if $\left\|h^{0.5} \hat{\varphi}\right\|_{H^{1}}$ is small, where $H^{1}=H^{1}(\Omega \times I)$ is the standard Hilbert space with associated norm. We note, however, that for turbulent flow the loss of sharpness in the bound in (7) may be significant, due to the use of a global Cauchy-Schwarz inequality. Therefore, in adaptive algorithms, the error representation in (5) is used directly, or with only local bounds on each cell in the finite element mesh. 


\subsection{Adaptive algorithms}

A posteriori error estimation provides a powerful tool for the development of robust and efficient adaptive algorithms. The basic idea of adaptive algorithms is to optimize the computational method with respect to the goal (output of interest) of the computation. Typical parameters of an adaptive finite element method include the local mesh size ( $h$-adaptivity), local degree of the finite element approximation ( $p$-adaptivity), local shape of the cells ( $r$-adaptivity), or combinations thereof.

To construct an adaptive DFS algorithm we compute a solution $\hat{U}$ for which we use the error representation (5) to estimate the output error $|M(\hat{u})-M(\hat{U})|$, with $\hat{u}$ any DFS solution computed on a finer mesh than $\hat{U}$. We can either choose $\hat{u}$ and $\hat{U}$ to be two DFS solutions computed on different meshes in the mesh hierarchy generated by the adaptive algorithm, or alternatively to consider $\hat{u}$ to be an ideal solution computed on a finest mesh possible in which case we use the approximation $u \approx U$ in the dual problem (6).

An adaptive algorithm is based on an estimate of the local contribution to the global error, which is obtained by splitting the error representation (5) into a sum of error indicators $\mathcal{E}_{n, K}$ over the cells $K$ in the discrete space mesh $\mathcal{T}^{h}$, and the time intervals $I_{n}=\left(t_{n-1}, t_{n}\right), n=1, \ldots, N$, that is

$$
M(\hat{u})-M(\hat{U})=\sum_{n=1}^{N} \sum_{K \in \mathcal{T}^{h}} \mathcal{E}_{n, K}=\sum_{n=1}^{N} \sum_{K \in \mathcal{T}^{h}}[R(u ; \hat{u})-R(U ; \hat{U}), \hat{\varphi}]_{n, K}
$$

with $[\cdot, \cdot]_{n, K}$ an $L_{2}\left(K \times I_{n}\right)$ inner product.

\subsection{Approximation of the dual solution}

There are several technical aspects with respect to how to apply the error representation formula (8) in an adaptive algorithm, in particular with regards to the approximation of $\hat{\varphi}$, the solution to the dual problem.

The dual problem is linear, but runs backward in time which is a challenge since the DFS velocities $u$ and $U$ act as coefficients in the dual problem and thus have to be stored as data. In practice, the dual problem is often solved by using a similar finite element method as used for the primal DFS problem, and the coefficients $u$ and $U$ can be interpolated in time to minimize data storage, with possibly a restart scheme to increase accuracy. Also, several different approaches are possible for how to apply the error representation formula (8) in practice [42], to retain sharpness and achieve maximal robustness.

When we use the approximation $u \approx U$ in the dual problem we introduce a linearization error which is hard to estimate a priori, since worst case estimates based on Gröwall's inequality grossly overestimate the effect [36]. In practice, computations on a sequence of meshes with different associated approximations $U$ provide an estimate of the asymptotic effect of this linearization error on the resulting a posteriori error estimates.

More specifically, the accuracy in the a posteriori error estimates depends on the accuracy in the approximation of the dual problem, which is affected by (i) perturbations in data and (ii) errors from computational approximation. As part of the adaptive algorithm, approximations of the dual problem are computed on a hierarchy of computational meshes, which can be used as a robustness test with respect to (i)-(ii), improving the reliability of the a posteriori error estimates.

\section{DFS implementation}

We now give a detailed presentation of one implementation of DFS, in the form of the $\mathrm{cG}(1) \mathrm{cG}(1)$ method with piecewise linear approximation in space and time over tetrahedral meshes. In Section 4 we recall validation studies of this method.

\subsection{The $c G(1) c G(1)$ method}

The $\mathrm{cG}(1) \mathrm{cG}(1)$ method is based on the continuous Galerkin method $\mathrm{cG}(1)$ in space and time. With cG(1) in time the trial functions are continuous piecewise linear and the test functions piecewise constant. $\mathrm{cG}(1)$ in space 
corresponds to both test functions and trial functions being continuous piecewise linear. Let $0=t_{0}<t_{1}<\cdots<$ $t_{N}=T$ be a sequence of discrete time steps with associated time intervals $I_{n}=\left(t_{n-1}, t_{n}\right)$ of length $k_{n}=t_{n}-t_{n-1}$ and space-time slabs $S_{n}=\Omega \times I_{n}$, and let $W \subset H^{1}(\Omega)$ be a finite element space consisting of continuous piecewise linear functions on a tetrahedral mesh $\mathcal{T}^{h}$ of mesh size $h(x)$ with $W_{0}$ the functions $v \in W$ satisfying the Dirichlet boundary condition $\left.v \cdot n\right|_{\Gamma}=0$.

We seek $\hat{U}=(U, P)$, continuous piecewise linear in space and time: For $n=1, \ldots, N$, find $\left(U^{n}, P^{n}\right) \equiv\left(U\left(t_{n}\right)\right.$, $\left.P\left(t_{n}\right)\right)$ with $U^{n} \in V_{0}^{n} \equiv\left[W_{0}\right]^{3}$ and $P^{n} \in W$, such that

$$
\begin{aligned}
& \left(\left(U^{n}-U^{n-1}\right) k_{n}^{-1}+\bar{U}^{n} \cdot \nabla \bar{U}^{n}, v\right)-\left(P^{n}, \nabla \cdot v\right) \\
& \quad+\left(\nabla \cdot \bar{U}^{n}, q\right)+S D_{\delta}^{n}\left(\bar{U}^{n}, P^{n} ; v, q\right)=0 \quad \forall \hat{v}=(v, q) \in V_{0}^{n} \times W^{n},
\end{aligned}
$$

where $\bar{U}^{n}=\frac{1}{2}\left(U^{n}+U^{n-1}\right)$ and $P^{n}$ are piecewise constant in time over $I_{n}$, with the stabilizing term

$$
S D_{\delta}^{n}\left(\bar{U}^{n}, P^{n} ; v, q\right) \equiv\left(\delta_{1}\left(\bar{U}^{n} \cdot \nabla \bar{U}^{n}+\nabla P^{n}\right), \bar{U}^{n} \cdot \nabla v+\nabla q\right)+\left(\delta_{2} \nabla \cdot \bar{U}^{n}, \nabla \cdot v\right),
$$

and

$$
(v, w)=\sum_{K \in \mathcal{T}^{h}} \int_{K} v \cdot w d x,
$$

with the stabilization parameters $\delta_{1}=\kappa_{1}\left(k_{n}^{-2}+\left|U^{n-1}\right|^{2} h^{-2}\right)^{-1 / 2}$ and $\delta_{2}=\kappa_{2} h\left|U^{n-1}\right|$, where $\kappa_{1}$ and $\kappa_{2}$ are positive constants of unit size. We choose a time step size $k_{n}=C_{C F L} \min _{x \in \Omega} h /\left|U^{n-1}\right|$, with $C_{C F L}$ a constant of unit size.

Remark 1. The cG(1) discretization in time does not allow for a fully consistent stabilization of the method, since the velocity test functions are constant in time. To make the method fully consistent while keeping a least squares stabilization of the residual, we would need to use test functions that are linear discontinuous in time, corresponding to a $\mathrm{cG}(1) \mathrm{dG}(1)$ method. The main motivation for using $\mathrm{cG}(1) \mathrm{cG}(1)$ is the reduction of the number of degrees of freedom by a factor 2 .

\section{2. $c G(1) c G(1)$ energy equation}

We recall that a $\mathrm{cG}(1) \mathrm{cG}(1)$ solution satisfies a local energy equation, with dissipation directly proportional to the local residual, with the proof given in [15]. We define a family of smooth positive test functions $\phi_{n}(x)$ for $n=1, \ldots, N$, with local compact support supp $\phi_{n} \subset \Omega$, and we define a piecewise constant function in time defined by $\phi(x, t)=\phi_{n}(x)$ for $t \in I_{n}$, normalized such that

$$
\sum_{n=1}^{N} \int_{\Omega} \phi_{n}(x) d x k_{n}=1 .
$$

Theorem 2. Noting that $\delta_{i} \leq C h$, for $i=1,2$, we have the following local energy estimate for $\mathrm{cG}(1) \mathrm{cG}(1)$ :

$$
\begin{aligned}
& \mid \sum_{n=1}^{N}\left[\int_{\Omega}\left(\frac{1}{2}\left(\left|U^{n}\right|^{2}-\left|U^{n-1}\right|^{2}\right) k_{n}^{-1}+\nabla \cdot\left(\bar{U}^{n}\left(\frac{1}{2}\left|\bar{U}^{n}\right|^{2}+P^{n}\right)\right)\right) \phi_{n} d x\right] k_{n} \\
& \quad+\sum_{n=1}^{N}\left[\int_{\Omega}\left(\delta_{1}\left|\bar{R}_{1}\left(\bar{U}^{n}, P^{n}\right)\right|^{2}+\delta_{2}\left|\bar{R}_{2}\left(\bar{U}^{n}\right)\right|^{2}\right) \phi_{n} d x\right] k_{n} \mid \\
& \leq C h_{\max , \phi, n}^{1 / 2}
\end{aligned}
$$

with $h_{\max , \phi, n} \equiv \max _{n: s u p p} \phi_{n} \neq \emptyset\left(\max _{x \in \text { supp } \phi_{n}} h(x)\right)$, and with the residuals $\bar{R}_{1}\left(\bar{U}^{n}, P^{n}\right)$ and $\bar{R}_{2}\left(\bar{U}^{n}\right)$ defined by

$$
\begin{aligned}
& \bar{R}_{1}(v, q)=\dot{v}+\bar{U}^{n} \cdot \nabla v+\nabla q, \\
& \bar{R}_{2}(v, q)=\nabla \cdot v,
\end{aligned}
$$

for $t \in I_{n}$. 
If we choose a test function $\phi$ with a suitable local support, and with $\phi_{j}=0$ for all $j \neq n$, Theorem 2 states that for $t \in I_{n}$, a cG(1)cG(1) solution up to the factor $C h_{\max , \phi, n}^{0.5}$ weakly satisfies (integrated against a smooth positive test function with local support) a local energy equation of the form:

$$
\frac{d}{d t}\left(\frac{1}{2}\left|U^{n}\right|^{2}\right)+\nabla \cdot\left(\bar{U}^{n}\left(\frac{1}{2}\left|\bar{U}^{n}\right|^{2}+P^{n}\right)\right)=-D_{h}^{n}(\hat{U}),
$$

with $D_{h}^{n}(\hat{U}) \equiv \delta_{1}\left|\bar{R}_{1}\left(\bar{U}^{n}, P^{n}\right)\right|^{2}+\delta_{2}\left|\bar{R}_{2}\left(\bar{U}^{n}\right)\right|^{2} \geq 0$, a residual based numerical dissipation, and with $\frac{d}{d t}\left(\frac{1}{2}\left|U^{n}\right|^{2}\right) \equiv$ $\frac{1}{2}\left(\left|U^{n}\right|^{2}-\left|U^{n-1}\right|^{2}\right) k_{n}^{-1}$.

This local energy equation connects to a dissipative weak Euler solution [33], with inertial energy dissipation resulting from local non-smoothness of the solution, and not from any viscosity. The dissipative term $D_{h}^{n}(\hat{U})$ in $(13)$ reflects local non-smoothness in the solution identified by the residuals, and is observed to be independent of $h$ under sufficient mesh resolution [12,15]

\subsection{A posteriori error analysis and an adaptive algorithm}

Using standard techniques of a posteriori error analysis, see e.g. [36], we can derive an a posteriori error estimate for $\mathrm{cG}(1) \mathrm{cG}(1)$.

Theorem 3. If $\hat{U}=(U, P)$ solves (9), $\hat{u}=(u, p)$ is a weak solution to (1), and $\hat{\varphi}=(\varphi, \theta)$ solves (6), then we have the following a posteriori error estimate for the output $M(\hat{U})=[\hat{U}, \hat{\psi}]$ with respect to the reference output $M(\hat{u})=[\hat{u}, \hat{\psi}]:$

$$
|M(\hat{u})-M(\hat{U})| \leq \sum_{n=1}^{N} \sum_{K \in \mathcal{T}^{h}} \mathcal{E}_{n, K}
$$

where

$$
\mathcal{E}_{n, K} \equiv \int_{I_{n}}\left|R_{1}(\hat{U})\right|_{K} \cdot \omega_{1} d t+\int_{I_{n}}\left|R_{2}(U)\right|_{K} \omega_{2} d t+\int_{I_{n}}\left|S D_{\delta}^{n}(\hat{U} ; \hat{\varphi})_{K}\right| d t
$$

with

$$
\begin{aligned}
& R_{1}(\hat{U})=\dot{U}+(U \cdot \nabla) U+\nabla P, \\
& R_{2}(U)=\nabla \cdot U,
\end{aligned}
$$

where $S D_{\delta}^{n}(\cdot ; \cdot)_{K}$ is a local version of the stabilizing form (10), and the stability weights are given by

$$
\begin{aligned}
& \omega_{1}=C_{n, K}^{k} k_{n}|\dot{\varphi}|_{K, \infty}+C_{n, K}^{h} h_{K}|\nabla \varphi|_{K}, \\
& \omega_{2}=C_{n, K}^{k} k_{n}|\dot{\theta}|_{K, \infty}+C_{n, K}^{h} h_{K}|\nabla \theta|_{K},
\end{aligned}
$$

where $h_{K}$ is the diameter of element $K$ in the mesh $\mathcal{T}^{h}, C_{n, K}^{h}, C_{n, K}^{k}$ represent interpolation constants, $|w|_{K} \equiv$ $\left(\left\|w_{1}\right\|_{K},\left\|w_{2}\right\|_{K},\left\|w_{3}\right\|_{K}\right)$ with $\|w\|_{K}=(w, w)_{K}^{1 / 2}$, and the dot denotes the scalar product in $\mathbb{R}^{3}$.

Based on the a posteriori error estimate (14), we design a basic adaptive algorithm where the mesh is kept constant in time. Starting from an initial mesh the primal problem is computed for the full time interval after which the dual problem is solved backwards over the same time interval using a similar $\mathrm{cG}(1) \mathrm{cG}(1)$ discretization as for the primal problem (same trial and test space, least squares stabilization of the residual except the time derivative, and the same stabilization parameters and time discretization). The error indicators $\mathcal{E}_{K}=\sum_{n=1}^{N} \mathcal{E}_{n, K}$ are then computed for each cell $K$ in the mesh. The cells with the highest error indicators $\mathcal{E}_{K}$ are bisected to generate a new refined mesh, for which the algorithm starts over until convergence in $M(\hat{U})$ is detected.

The primal solution $U$ for each mesh is stored to be used as data for the dual problem, where the approximation $u \approx U$ is used for the coefficient $u$ in (6). For demanding large scale problems where it is too expensive to store the complete time series of the primal solution, snapshots of $\hat{U}$ are stored with a certain frequency, and intermediate solution fields are reconstructed by interpolation in time. 
Adaptive algorithm: given initial coarse mesh $\mathcal{T}_{0}^{h}, k=0$,

(1) compute the solution $\hat{U}$ to the primal problem using $\mathcal{T}_{k}^{h}$

(2) compute the solution $\hat{\varphi}$ to the dual problem using $\mathcal{T}_{k}^{h}$

(3) if convergence in $M(\hat{U})$ is observed, or if the estimated error $\sum_{K \in \mathcal{T}^{h}} \mathcal{E}_{K}$ is less than a given tolerance, then STOP, else

(4) bisect $10 \%$ of cells $K \in \mathcal{T}_{k}^{h}$ with highest error indicators $\mathcal{E}_{K}$

(5) set $k=k+1$, then goto (1)

\subsection{Software implementation}

The adaptive algorithm and the $\mathrm{cG}(1) \mathrm{cG}(1)$ solvers for the primal and dual problems are implemented in the continuum mechanics solver Unicorn [43] that builds on the high performance (HPC) branch of the finite element problem solving environment DOLFIN, part of the FEniCS project [44]. DOLFIN-HPC is optimized for distributed memory architectures using a hybrid MPI+OpenMP approach with efficient parallel I/O (MPI I/O) [45,46], where ParMETIS [47] is used for mesh partitioning. DOLFIN-HPC supports several parallel linear algebra packages, but mostly rely on PETSc [48].

\section{Validation and application of DFS}

DFS is based on the computation of finite element approximations on adaptively refined computational meshes. In particular, no ad hoc mesh design is needed, since solutions features such as flow separation and turbulent wakes are automatically detected by the adaptive method through a posteriori error estimation with sensitivity information from the solution of a dual problem. A hierarchy of adaptively refined meshes is generated for which convergence can be observed with respect to an output of interest. The method has been applied for a number of bluff body flow problems over a range of Reynolds numbers, and we here recall some validation results for DFS in the form of the cG(1)cG(1) method.

\subsection{Medium Reynolds number flow}

At medium Reynolds numbers, $\mathrm{Re}<10^{5}$, boundary layers are laminar and can be resolved by the computational mesh. For this case, viscous effects are not negligible so that the viscosity is kept in the model (1), and no slip boundary conditions are chosen where the velocity is set to zero on the solid boundary $\Gamma$. DFS in the form of the cG(1)cG(1) method has been validated for a number of model problems of simple geometry bluff bodies, including a surface mounted cube and a rectangular cylinder [12,11], a sphere [13] and a circular cylinder [14]. In each case, convergence is observed for output quantities such as drag, lift and pressure coefficients, and Strouhal numbers, and the adaptive algorithm leads to an efficient method often using orders of magnitude fewer number of degrees of freedom compared to LES methods based on ad hoc design of the mesh.

\subsection{High Reynolds number flow}

In high Reynolds number flow, with $R e>10^{6}$, boundary layers are turbulent and are in most cases too expensive to resolve, and must instead be modeled. To accurately predict e.g. aerodynamic forces it is critical to capture the correct flow separation, which can be connected to the boundary layer, in case flow separation is not triggered by sharp features in the geometry [15]. Drag crisis for a circular cylinder is an illustrative example, where flow separation moves downstream the cylinder wall as a consequence of transition to turbulence in the boundary layer [49]. In [18] we model drag crisis by decreasing the skin friction parameter $\beta$ in the model (1), where we find that the DFS simulations reproduce the observed drag crisis scenario from experiments [50-53].

In particular, we find that for small $\beta$ the solution is insensitive to the particular value of $\beta$, so that we reach an ultimate regime where the turbulent boundary layer is modeled by a zero friction free slip boundary condition. This free slip model has the benefit of being a parameter-free model of turbulent boundary layers, which requires no boundary layer mesh since no boundary layer is resolved. The basic assumptions underlying the model is that the 
turbulent boundary layers are fully developed, since transition to turbulence in the boundary layers is not part of the model.

For a high Reynolds number, the free slip boundary condition has been shown to be a good model in the sense that flow separation and thereby the surface pressure distribution is well approximated. For high Reynolds numbers, the main contribution to the aerodynamic forces on a body comes from the surface pressure distribution, and thus the free slip model has a high potential in aerodynamics applications.

DFS with a free slip boundary condition has been validated for the standard benchmarks of a NACA 0012 wing [54] and a 30P30N high lift device [4], where close agreement with experimental measurement was found in aerodynamic forces and surface pressure distribution.

\subsection{Complex geometry}

To assess the capability of DFS for challenging high Reynolds number flow problems in complex geometry, we have participated in a number of benchmark workshops with detailed experimental data available for validation, including the first, second and third Workshop on Benchmark problems for Airframe Noise Computations (BANC-I, -II, -III) [2-4], and the 2nd AIAA CFD High Lift Prediction Workshop (HiLiftPW-2) [5].

Good agreement was found between DFS simulations and experimental measurements in all workshops, and two features distinguished DFS from all other methodologies: (i) DFS was the only method using adaptive mesh optimization, and (ii) DFS was the only method leaving boundary layers unresolved (free slip boundary conditions were used for all problems). Both (i) and (ii) contributed to the fact that the number of degrees of freedom used in DFS was significantly lower than in other methods, often several magnitudes less.

In HiLiftPW-2 the task was to simulate flow at high Reynolds number around a full wing-body model of an airplane, where DFS was the only method based on the Navier-Stokes equations that simulated the unsteady flow [5]; aside from 2 contributions based on Lattice Boltzmann Methods all other contributions used stationary RANS. Good agreement of DFS simulations and experimental data was found in aerodynamic forces and surface pressure distributions on the wings, and the transient simulation data provided additional data on the flow at high angles of attack modeling take-off and landing. In particular, convergence of the adaptive algorithm to experimental reference data for lift and drag was observed for angles of attack in the pre-stalled regime, see Fig. 1.

We note that since viscosity and skin friction are assumed to be negligible, the only input data to the DFS model is the geometry of the airplane, in the form of an initial coarse mesh.

\section{Discussion}

To our best knowledge, application of adjoint based adaptive methods to simulate unsteady turbulent flow in 3D is still rare [55], probably one of the main reasons is the challenges posed by the dual problem. In particular, there is a computational challenge associated with storing the primal solution used as data in the dual problem. To address this challenge for large problems, snapshots of the full spatial field $U$ can be stored with a certain frequency, from which intermediate fields can be reconstructed by interpolation, or by re-computing the primal solution over the intermediate time intervals.

Another challenge concerns the stability of the dual problem linearized at a turbulent flow field. Characteristic for turbulent flow are chaotic particle paths which make prediction of point values hard or impossible, popularly referred to as the butterfly effect. But turbulence is also a dissipative mechanism, similar to a shock in high Mach number flow, with many stable features in the sense of the macroscopic flow, which makes prediction of mean values possible. For example, although the particular set of particle paths around an airplane is impossible to predict, the net effect in terms of the time average of aerodynamic forces is a stable quantity.

\subsection{Stability of the dual problem}

Particle paths in a turbulence simulation are chaotic, but mean value output can be stable. The difference between two computed solutions $\hat{u}$ and $\hat{U}$ with respect to an output functional $M(\cdot)$ can be expressed as the duality pairing of local residual errors with the solution of a dual (adjoint) problem linearized at the two computed solutions, which is the posteriori error representation (5) that is the foundation of the DFS adaptive method. In particular, the computational 

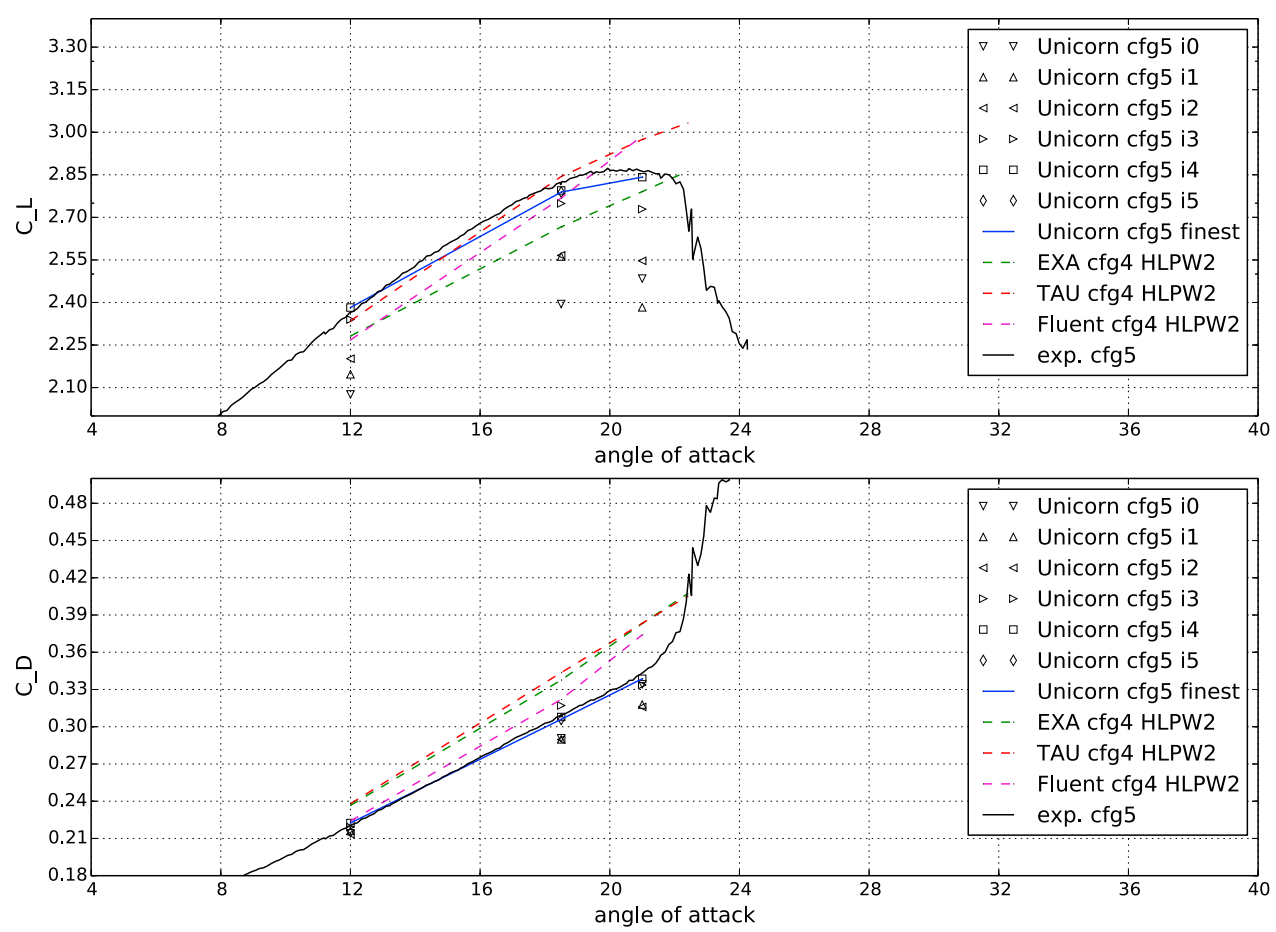

Fig. 1. Convergence under adaptive mesh refinement of drag and lift coefficients towards experimental values (Unicorn) (results in preparation to be published), compared to state-of-the-art CFD methods (EXA, TAU, Fluent) (published as part of the HighLiftPW-2 workshop).
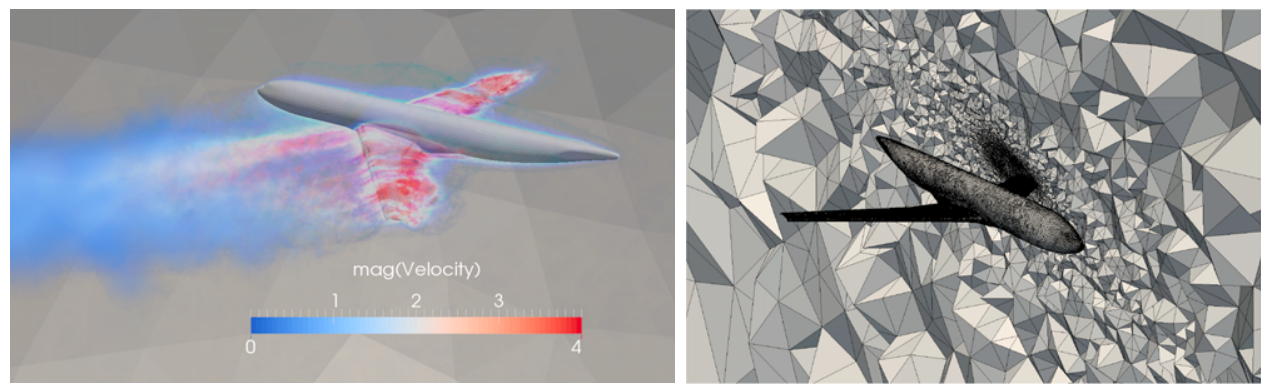

Fig. 2. From [5]: Snapshot of the magnitude of dual velocity (left), and an adaptively refined computational mesh optimized for lift and drag approximation (right).

approximation of the dual solution is at the heart of the methodology, and thus the well-posedness of the dual problem is critical: is the dual problem solvable, and what is the sensitivity in the dual solution with respect to its data (the linearization error)?

A worst case analytical estimate using Grönwall's inequality bounds the dual solution in terms of rapid exponential growth in time [36], although in practice approximations of the dual solution for turbulent flow are routinely computed as part of the DFS method, see e.g. Fig. 2. Practical computation also shows that the dual solution is stable with respect to perturbations in data in the form of approximate primal solutions on different computational meshes. That is, when the computational mesh is sufficiently refined so that the output functional of interest $M(\hat{U})$ (e.g. drag) has converged, little difference is seen in dual solutions $\hat{\varphi}$ linearized at different approximate solutions $\hat{U}$ from this class of approximate solutions, which is observed as part of the adaptive algorithm [36].

The dual equations is a system of convection-reaction equations that take the gradient $\nabla U$ as reaction coefficient. Given the sharp gradients in turbulent flow, how is well-posedness of the dual problem possible? We observe computability of the discretized dual equations, but from this we cannot infer that the continuous dual problem is stable. In [36] we argue that the stability of the dual problem is possible by cancellation, since in a turbulent flow the gradient 

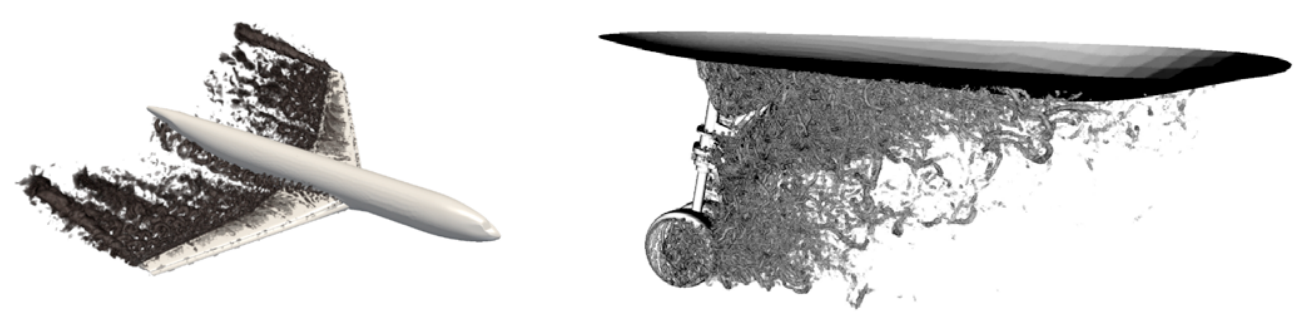

Fig. 3. Vorticity visualized by a Q-criterion: snapshot of a DFS solution of the flow around of a wing-body configuration (left) [5], and flow around a Gulfstream G550 nose landing gear (right) [3].

is oscillating rapidly, so that exponential growth is alternating with exponential decay. In particular, a turbulent wake is dominated by vortices on a range of scales, see Fig. 3, where each individual vortex tube is a stable flow structure in the sense that a linearized stability analysis show no exponential perturbation growth [36].

\subsection{Blowup of the dual solution}

Exponential growth of the dual solution with drag data is reported in [56] for flow around a cylinder at $R e=500$, which conforms with worst case analytical estimates. This is contrary to the observations we have made for bluff body flow at higher Reynolds numbers, reported e.g. in [14] for circular cylinder flow at $R e=3900$, where the dual solution is stable with converging a posteriori error estimates.

Is this difference related to differences in the computational methods used to compute the approximate dual solutions, or is it related to a fundamental difference between the two flows? This is still an open question, but we note that the flow at $R e=500$ and $R e=3900$ have important differences, where the flow at $R e=500$ has a more pronounced von Karman street whereas the turbulent wake is more stable at $R e=3900$.

If dual solutions consistently would show blowup for high Reynolds number turbulent flow, then the linearized approach can be questioned for this class of flows. One alternative to the linearized error analysis would be forward propagation of perturbations through ensembles of solutions. For example, in weather forecasting both these approaches are used to quantify uncertainties in the predictions.

Whether there is a fundamental problem with duality based methods for turbulent flow remains to be seen, but so far we have not encountered any stability problems using the methods presented in this paper, other than for the case of 2D compressible flow at increasing Reynolds numbers [57], where strong vorticity production in the primal problem is the result of the sharpening of boundary layers, and where no non-viscous mechanism for dissipation of vorticity is available in 2D. In 3D flow, turbulence develop that dissipate vorticity through vortex stretching, which can be observed in corresponding 3D simulations [14].

\section{Summary}

We have reviewed a computational framework for turbulence simulation based on adaptive finite element approximation, which we refer to as Direct Finite Element Simulation (DFS). For high Reynolds numbers, viscosity and wall shear stress are assumed to be negligible compared to inertial effects, and thus the DFS model has no empirical parameters. We interpret a DFS approximation as a dissipative weak solution, for which we can estimate the error with respect to output functionals using duality analysis.

Validation of the method has been carried out for a number of benchmark problems, where it is found that DFS simulations compare well with experimental data, while at the same time being a very efficient methodology in terms of the number of degrees of freedom needed to compute output data such as aerodynamics forces and surface pressure distributions. The main distinguishing features of the parameter-free version of DFS are: (i) the computational mesh is optimized based on a goal functional using a posteriori error estimation, and (ii) turbulent boundary layers are left unresolved.

\section{Acknowledgments}

The authors acknowledge the financial support from the Swedish Foundation for Strategic Research, the European Research Council (Grant no. 202984), and the Swedish Research Council (ID 90032202). The simulations were per- 
formed on resources provided by the Swedish National Infrastructure for Computing (SNIC) at PDC - Center for High-Performance Computing, and the Barcelona Supercomputing Center - Centro Nacional de Supercomputacion. The author would like to acknowledge the financial support by the Basque Excellence Research Center (BERC 20142017) program by the Basque Government, the Spanish Ministry of Economy and Competitiveness MINECO: BCAM Severo Ochoa accreditation SEV-2013-0323 and the Project of the Spanish Ministry of Economy and Competitiveness with reference MTM2013-40824.

\section{References}

[1] R.V. Abreu, N. Jansson, J. Hoffman, Adaptive computation of aeroacoustic sources for a rudimentary landing gear, Internat. J. Numer. Methods Fluids 74 (6) (2014) 406-421.

[2] R.V. de Abreu, N. Jansson, J. Hoffman, Adaptive computation of aeroacoustic sources for a rudimentary landing gear using lighthills analogy, AIAA Paper 2942 (2011) 2011.

[3] R.V. de Abreu, N. Jansson, J. Hoffman, Computation of aeroacoustic sources for a complex nose landing gear geometry using adaptivity, in: Proceedings of the Second Workshop on Benchmark problems for Airframe Noise Computations, BANC-II, Colorado Springs, 2012.

[4] J. Hoffman, J. Jansson, R.V. de Abreu, Computation of slat noise sources using adaptive fem and lighthills analogy, in: 19th AIAA/CEAS Aeroacoustics Conference, 2013.

[5] J. Hoffman, J. Jansson, N. Jansson, R.V. de Abreu, Time-resolved adaptive fem simulation of the dlr-f11 aircraft model at high Reynolds number, in: AIAA 2014-0917, Proc. 52nd Aerospace Sciences Meeting, AIAA SciTech, 2014.

[6] K. Eriksson, D. Estep, P. Hansbo, C. Johnson, Introduction to adaptive methods for differential equations, Acta Numer. 4 (1) (1995) $105-158$.

[7] J.T. Oden, S. Prudhomme, Goal-oriented error estimation and adaptivity for the finite element method, Comput. Math. Appl. 41 (5-6) (2001) $735-756$.

[8] R. Becker, R. Rannacher, An optimal control approach to a posteriori error estimation in finite element methods, Acta Numer. 2001 (10) (2001) 1-102.

[9] J. Hoffman, C. Johnson, Adaptive finite element methods for incompressible fluid flow, in: Error Estimation and Adaptive Discretization Methods in Computational Fluid Dynamics, Springer, 2003, pp. 97-157.

[10] J. Hoffman, On duality-based a posteriori error estimation in various norms and linear functionals for large eddy simulation, SIAM J. Sci. Comput. 26 (1) (2004) 178-195

[11] J. Hoffman, C. Johnson, A new approach to computational turbulence modeling, Comput. Methods Appl. Mech. Engrg. 195 (23) (2006) $2865-2880$.

[12] J. Hoffman, Computation of mean drag for bluff body problems using adaptive dns/les, SIAM J. Sci. Comput. 27 (1) (2005) $184-207$.

[13] J. Hoffman, Adaptive simulation of the subcritical flow past a sphere, J. Fluid Mech. 568 (2006) 77-88.

[14] J. Hoffman, Efficient computation of mean drag for the subcritical flow past a circular cylinder using general galerkin g2, Internat. J. Numer. Methods Fluids 59 (11) (2009) 1241-1258.

[15] J. Hoffman, J. Jansson, R. Vilela De Abreu, Adaptive modeling of turbulent flow with residual based turbulent kinetic energy dissipation, Comput. Methods Appl. Mech. Engrg. 200 (37) (2011) 2758-2767.

[16] P. Sagaut, Large Eddy Simulation for Incompressible Flows, third ed., Springer, 2005.

[17] J. Hoffman, Simulation of turbulent flow past bluff bodies on coarse meshes using general galerkin methods: drag crisis and turbulent euler solutions, Comput. Mech. 38 (4-5) (2006) 390-402.

[18] J. Hoffman, N. Jansson, A computational study of turbulent flow separation for a circular cylinder using skin friction boundary conditions, in: Quality and Reliability of Large-Eddy Simulations II, Springer, 2011, pp. 57-68.

[19] J. Hoffman, C. Johnson, Resolution of dalemberts paradox, J. Math. Fluid Mech. 12 (3) (2010) 321-334.

[20] U. Piomelli, E. Balaras, Wall-layer models for large-eddy simulations, Annu. Rev. Fluid Mech. 34 (1) (2002) 349-374.

[21] J. Smagorinsky, General circulation experiments with the primitive equations: I. the basic experiment, Mon. Weather Rev. 91 (3) (1963) 99-164.

[22] J. VonNeumann, R.D. Richtmyer, A method for the numerical calculation of hydrodynamic shocks, J. Appl. Phys. 21 (3) (1950) $232-237$. URL http://scitation.aip.org/content/aip/journal/jap/21/3/10.1063/1.1699639.

[23] C. Fureby, F. Grinstein, Monotonically integrated large eddy simulation of free shear flows, AIAA J. 37 (5) (1999) $544-556$.

[24] Y. Bazilevs, V. Calo, J. Cottrell, T. Hughes, A. Reali, G. Scovazzi, Variational multiscale residual-based turbulence modeling for large eddy simulation of incompressible flows, Comput. Methods Appl. Mech. Engrg. 197 (1) (2007) 173-201.

[25] J. Principe, R. Codina, F. Henke, The dissipative structure of variational multiscale methods for incompressible flows, Comput. Methods Appl. Mech. Engrg. 199 (13) (2010) 791-801.

[26] J. Meyers, B. Geurts, P. Sagaut, A computational error-assessment of central finite-volume discretizations in large-eddy simulation using a smagorinsky model, J. Comput. Phys. 227 (1) (2007) 156-173.

[27] J. Leray, Sur le mouvement d'un liquide visqueux emplissant l'espace, Acta Math. 63 (1) (1934) 193-248.

[28] J. Guermond, J. Oden, S. Prudhomme, An interpretation of the Navier-Stokes-alpha model as a frame-indifferent leray regularization, Physica D 177 (1) (2003) 23-30.

[29] C. Fefferman, Official clay prize problem description: existence and smoothness of the Navier-Stokes equation (2000).

[30] V. Scheffer, et al., Partial regularity of solutions to the Navier-Stokes equations, Pacific J. Math. 66 (2) (1976) 535-552.

[31] L. Caffarelli, R. Kohn, L. Nirenberg, Partial regularity of suitable weak solutions of the Navier-Stokes equations, Comm. Pure. Appl. Math. 35 (6) (1982) 771-831.

[32] J.-L. Guermond, On the use of the notion of suitable weak solutions in cfd, Internat. J. Numer. Methods Fluids 57 (9) (2008) $1153-1170$. 
[33] J. Duchon, R. Robert, Inertial energy dissipation for weak solutions of incompressible euler and Navier-Stokes equations, Nonlinearity 13 (1) (2000) 249.

[34] L. Onsager, Statistical hydrodynamics, Il Nuovo Cimento 1943-1954 (6) (1949) 279-287.

[35] G.L. Eyink, K.R. Sreenivasan, Onsager and the theory of hydrodynamic turbulence, Rev. Modern Phys. 78 (1) (2006) 87.

[36] J. Hoffman, C. Johnson, Computational Turbulent Incompressible Flow: Applied Mathematics: Body and Soul 4, Vol. 4, Springer, 2007.

[37] J. Hoffman, C. Johnson, Blow up of incompressible euler solutions, BIT 48 (2) (2008) 285-307.

[38] P.R. Spalart, Detached-eddy simulation, Annu. Rev. Fluid Mech. 41 (2009) 181-202.

[39] H. Schlichting, K. Gersten, K. Gersten, Boundary-layer Theory, Springer, 2000.

[40] P. Moin, J. Kim, Tackling turbulence with supercomputers, Sci. Am. 276 (1) (1997) 46-52.

[41] L. Prandtl, On fluid motions with very small friction, in: Verhldg. 3 Int. Math. Kongress, Wiley, 1904, pp. 484-491.

[42] W. Bangerth, R. Rannacher, Adaptive Finite Element Methods for Differential Equations, Springer, 2003.

[43] J. Hoffman, J. Jansson, R. Vilela de Abreu, N.C. Degirmenci, N. Jansson, K. Müller, M. Nazarov, J.H. Spühler, Unicorn: parallel adaptive finite element simulation of turbulent flow and fluid-structure interaction for deforming domains and complex geometry, Comput. \& Fluids 80 (2013) 310-319.

[44] The fenics project.

[45] N. Jansson, J. Hoffman, J. Jansson, Framework for massively parallel adaptive finite element computational fluid dynamics on tetrahedral meshes, SIAM J. Sci. Comput. 34 (1) (2012) C24-C41.

[46] N. Jansson, J. Hoffman, M. Nazarov, Adaptive simulation of turbulent flow past a full car model, in: 2011 International Conference for High Performance Computing, Networking, Storage and Analysis, SC, IEEE, 2011, pp. 1-8.

[47] Parmetis - parallel graph partitioning and fill-reducing matrix ordering.

[48] Petsc — portable, extensible toolkit for scientific computation.

[49] M. Zdravkovich, Flow around circular cylinders, vol. 1. fundamentals, J. Fluid Mech. 350 (1997) 377-378.

[50] A. Korotkin, The three-dimensional character of a cross flow around a circular cylinder, TsAGI, Uchenye Zap. 4 (5) (1973) $26-33$.

[51] J.S. Humphreys, On a circular cylinder in a steady wind at transition Reynolds numbers, J. Fluid Mech. 9 (04) (1960) 603-612.

[52] B. Gölling, Experimentelle untersuchungen des laminar-turbulenten überganges der zylindergrenzschichtströmung (Ph.D. thesis), DLR, 2001.

[53] G. Schewe, Reynolds-number effects in flow around more-or-less bluff bodies, J. Wind Eng. Ind. Aerodyn. 89 (14) (2001) $1267-1289$.

[54] J. Jansson, J. Hoffman, N. Jansson, Simulation of 3d unsteady incompressible flow past a naca 0012 wing section, CTL Technical Report kth-ctl-4023.

[55] K.J. Fidkowski, D.L. Darmofal, Review of output-based error estimation and mesh adaptation in computational fluid dynamics, AIAA J. 49 (4) (2011) 673-694.

[56] Q. Wang, J.-H. Gao, The drag-adjoint field of a circular cylinder wake at Reynolds numbers 20, 100 and 500, J. Fluid Mech. 730 (2013) $145-161$.

[57] M. Nazarov, J. Hoffman, On the stability of the dual problem for high Reynolds number flow past a circular cylinder in two dimensions, SIAM J. Sci. Comput. 34 (4) (2012) A1905-A1924. 